\title{
Evaluations of Elliptical Modulation Scheme
}

\author{
Chunyi Song, Shigeru Shimamoto \\ Graduate School of Global Information and Telecommunication Studies \\ Waseda University \\ Bldg. 29-7, 1-3-10 Nishi-Waseda, Shinjuku-ku, Tokyo 169-0051, Japan \\ song@sl.giti.waseda.ac.jp shima@giti.waseda.ac.jp
}

\begin{abstract}
This paper aimed to evaluate some modulation methods, which have been defined based on Elliptical Modulation Scheme, namely are Eccentricity Shift Keying (ESK), Inclination Angle Shift Keying (IASK) and Elliptical Phase Shift Keying (EPSK). In this paper, signal characteristics of these three modulation schemes and effect of eccentricity to the waveforms have been demonstrated and analysed, through observing real signals generated by using DSPs, both in time-domain and frequency-domain; performances of IASK, 4-EPSK and 8-EPSK, as three modulation methods capable of 1-bit, 2-bit and 3-bit information transmission under the same carrier frequency respectively, have been evaluated through Matlab simulation in terms of BER, advantages over existing modulation schemes suggested that newly proposed modulation schemes can contribute to the improvement of wide-band wireless communication systems.
\end{abstract}

Keywords-elliptical modulation; DSPs; BER; characteristics

\section{INTRODUCTION}

In wireless communication system, most of existing modulation methods modulates signals by using sine waves derived from a circle, and there are only three characteristics of a signal that can be changed over time, which are amplitude, frequency and phase. By employing geometrical characteristics of an ellipse to manipulate a signal, Elliptical Modulation, which has been proposed by us before [1], introduces additional attributes such as eccentricity, offset inclination angle, rotation frequency and rotation direction. Introduction of new modulation variables provides Elliptical Modulation Scheme with higher flexibility, which implies that quite a lot of modulation methods can be developed through combining all kinds of modulation parameters, among which some may contribute to the improvement of wireless communication systems based on different advantages over existing modulation scheme.

In section II, definitions of some modulation methods based on Elliptical Modulation were reviewed at first, which are Eccentricity Shift Keying (ESK), Inclination Angle Shift Keying (IASK) and Elliptical Phase Shift Keying (EPSK). In section III, signal characteristics of these three modulation schemes, and effects of eccentricity to the waveforms have been demonstrated and analysed, through observing real signals generated by using DSPs, both in time-domain and frequency-domain. BER performances of IASK, 4-EPSK and 8-EPSK were evaluated through Matlab simulation in section IV, effect of eccentricity to the detection result has also been investigated in IASK simulation. Finally the paper was concluded in section $\mathrm{V}$.

\section{REVIEWS OF ELLIPTICAL MODULATION}

In this section, definitions of ESK, IASK and EPSK were reviewed. Expressions of the modulated signals given out in each scheme are coherent to those used to generate real signals in section 3 .

\section{A. Equation of ellipse in polar coordinates}

General equation of ellipse in polar coordinates can be expressed as:

$$
r=a \sqrt{\frac{1-e^{2}}{1-e^{2} \cos ^{2}(\theta+\alpha)}}
$$

Where $\quad \mathrm{r}=$ radius, $\mathrm{a}=$ semi-major axis, $\mathrm{e}=$ eccentricity, $\theta=$ revolution angle, and $\alpha=$ offset inclination angle.

\section{B. Eccentricity Shift Keying (ESK)}

ESK signals can be expressed as:

$$
s(t)=a \sqrt{\frac{1-e_{i}^{2}}{1-e_{i}^{2} \cos ^{2}\left(\omega_{r} t+\alpha\right)}} \cos \omega_{r} t \quad i=1,2, . . M
$$

Where a=semi-major axis, $e_{i}=$ eccentricity, $\omega_{\mathrm{r}}=$ revolution angular frequency, $\alpha=$ offset inclination angle.

Equation (2) implies that change of eccentricities can give rise to different waveforms, thus binary numbers 0 and 1 can be assigned to two ellipses of having different eccentricities. This is defined as ESK.

\section{Inclination Angle Shift Keying (IASK)}

Distinctive waveforms can be produced by changing offset inclination angles (Fig.1), which suggests that binary numbers 0 and 1 can be represented by two ellipses inclined at different angles. This is defined as IASK. For instance, two ellipses inclined at $\pi / 4$ and $-\pi / 4$ can be used to represent binary numbers 0 and 1 respectively.

IASK signals are expressed as: 


$$
s(t)=a \sqrt{\frac{1-e_{c}^{2}}{1-e_{c}^{2} \cos ^{2}\left(\omega_{r} t+\alpha_{i}\right)}} \cos \omega_{r} t \quad i=1,2, . . M
$$

Where $\alpha_{i}=$ offset inclination angle, $\mathrm{e}_{\mathrm{c}}=$ eccentricity, $\omega_{\mathrm{r}}=$ revolution angular frequency.

\section{Elliptical Phase Shift Keying}

Definition of EPSK is accomplished through combining effect of offset inclination angle and that of signal's phase. General equation of EPSK signals can be given out as:

$$
s(t)=a \sqrt{\frac{1-e_{c}^{2}}{1-e_{c}^{2} \cos ^{2}\left(\omega_{r} t+\alpha_{i}\right)}} \cos \left(\omega_{r} t+\phi_{j}\right)
$$

Where $\alpha_{i}$ and $\phi_{j}$ can have $\mathrm{N}$ and $\mathrm{M}$ discrete values respectively, and thus transmission capacity of EPSK is determined by the possible combination number of $\mathrm{N} \times \mathrm{M}$;

As examples, when both $N$ and $M$ were fixed to value " 2 ", for its capability of 2-bit information transmission under the same carrier frequency, Four-Elliptical Phase Shift Keying (4-EPSK) was defined through combining BPSK based on elliptical carrier and IASK; While in the case when $N$ and $M$ were fixed to "2" and "4" respectively, 8-EPSK was defined through combining QPSK based on elliptical carrier and IASK, as a method capable of transmitting 3-bit information under the same carrier frequency.

Fig. 2 is the constellations of 8-EPSK, as has been done in IASK, ellipses inclined at $\pi / 4$ and $-\pi / 4$ can be used to represent the first bit of binary numbers 0 and 1 (the bold numbers) respectively. Message sequences of the last two bits $00 \sim 11$ then can be assigned to four phases in four quadrants of each constellation. Thus, all 3-bit message sequences 000 111 have been mapped to the two constellations.

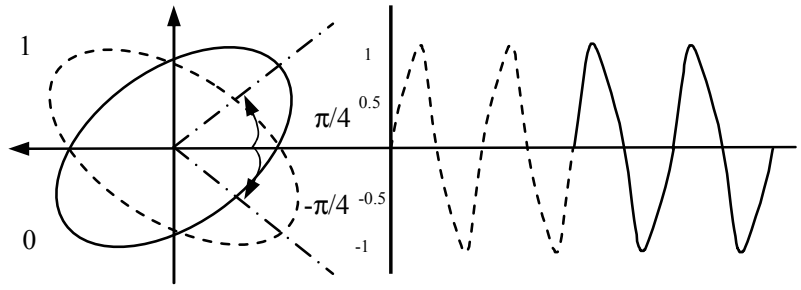

Figure 1. Example of IASK signals

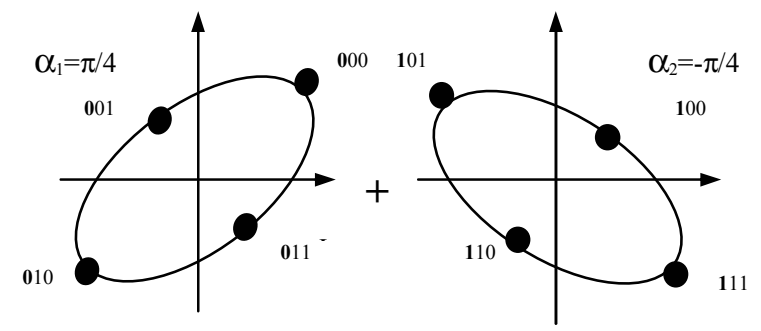

Figure 2. Constellations of 8-EPSK

\section{SIGNAL CHARACTERISTICS OF ELLIPTICAL MODULATION SCHEME}

In this section, real signals generated by using DSPs have been observed and analysed both in time-domain and frequency-domain. Characteristics of the signals, especially effects of eccentricity to the waveforms and power spectrums shown in the results, can be helpful in deciding the optimum value of the modulation variables to achieve excellent performance in simulation.

\section{A. Eccentricity Shift Keying}

Combination of eccentricity and semi-major axis decides the shape of an ellipse. When eccentricity equals to zero, the ellipse becomes a circle, and a circle produces a pure sine wave. Signals were observed to appear as pure sine waves at eccentricities below 0.4 , distinctions among the waveforms can only be made on the power output of each waveform. As the eccentricity was increased from 0.6 to 0.95 , waveforms become more distinctive with the increase in amplitude (Fig.3). Therefore, signals with larger eccentricities are predicted to be appeared with more frequency components in frequencydomain.

As we know, a pure sine wave derived from a circle has no harmonic distortion. Spectrum analyzer showed that signals of having eccentricities below 0.4 were also made up of several frequencies, although they appeared to be pure sine waves in time-domain - what was indiscernible on the Oscilloscope became very distinguishable on the Spectrum Analyzer.

For ellipses of having eccentricities 0.2 and 0.4 , only one harmonic appeared in the power spectrum. As eccentricity was increased from 0.6 to 0.9 , more harmonics were observed. Amplitude of main lobe was set to be 0 , and then relative amplitudes of side-lobes, or say harmonic distortions, were recorded and summarized in Fig.4. Increase in eccentricity of ESK signal give rise to the increase in number of harmonic distortions, and the increase in powers of the side lobes.

\section{B. Inclination Angle Shift Keying}

As can be seen from ESK signals shown in Fig.3, Eccentricity has apparent effect to the shape of elliptical waveform. Here $\pm \pi / 4$ IASK waveforms were observed by using Wave Runner at three different eccentricities: 0.3, 0.6 and 0.9 (Fig. 5 \& Fig. 6).

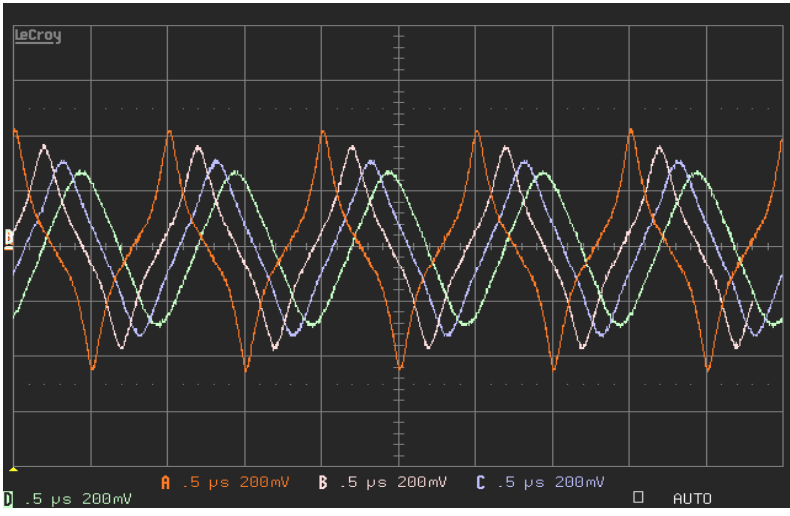

Figure 3. ESK signals at eccentricities of 0.6, 0.8, 0.9 and 0.95 


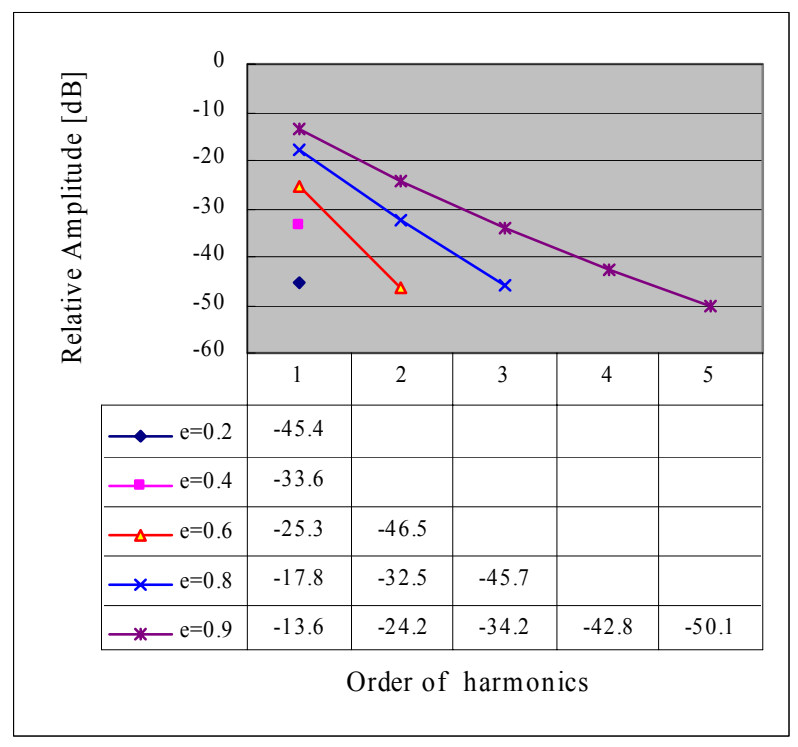

Figure 4. ESK Spectrum Analysis

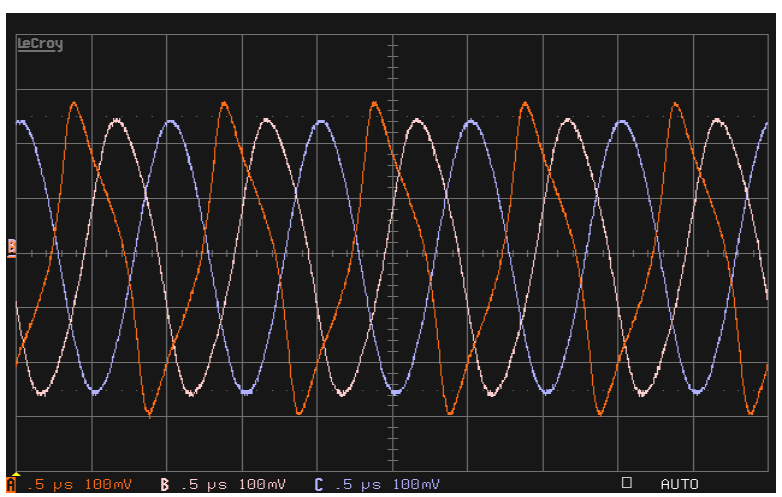

Figure 5. $\pi / 4$ IASK signals at eccentricities of $0.3,0.6$ and 0.9

As eccentricity was increased from 0.3 to 0.6 , no significant difference but only slight increase in the amplitude was observed in the shape of the waveforms. At eccentricity 0.9, waveform of the elliptical carrier inclined at $\pi / 4$ leans to the left, while it leans to the right when the ellipse was set to incline at $-\pi / 4$. This distinction might be employed to distinguish the two signals from each other.

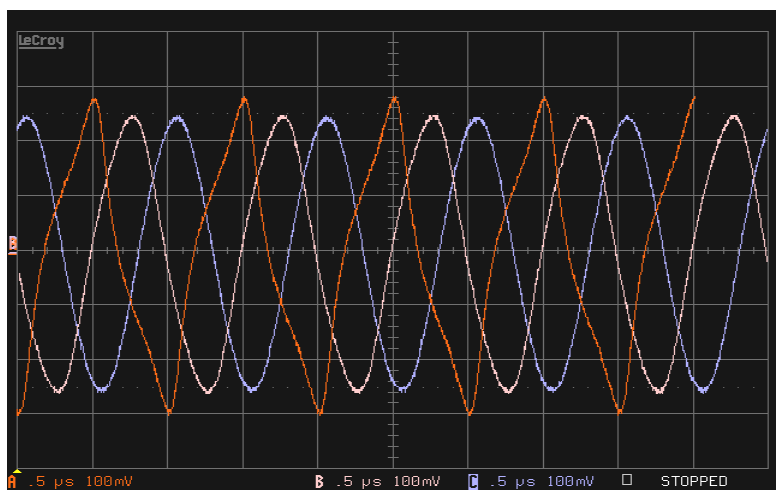

Figure $6 . \quad-\pi / 4$ IASK signals at eccentricities of $0.3,0.6$ and 0.9

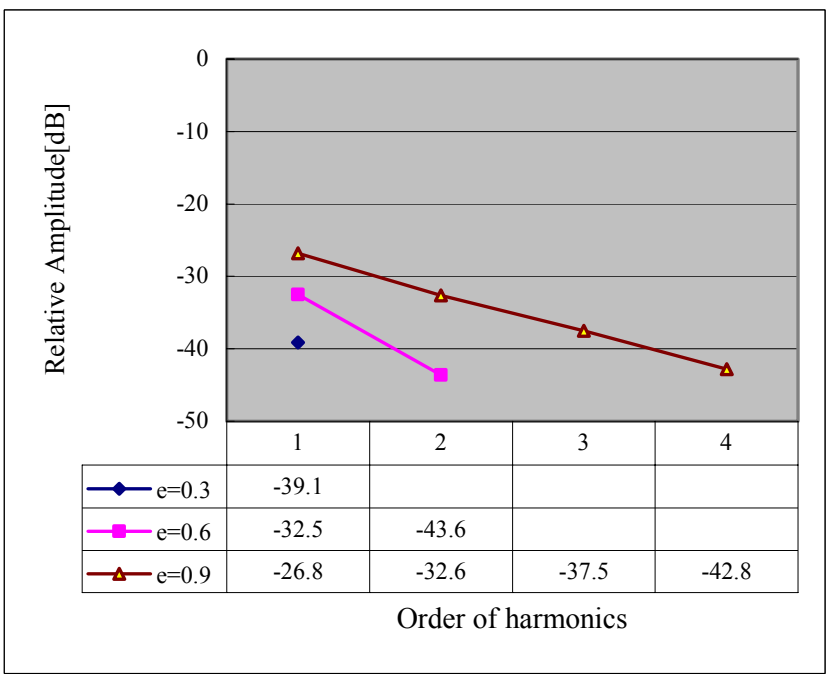

Figure 7. IASK Spectrum Analysis

When being viewed under the Spectrum Analyzer, display showed that more and more harmonics appeared as eccentricity was increased from 0.3 to 0.6 , and then to 0.9 . Relative amplitudes of side-lobes, or say harmonic distortions, were recorded and summarized in Fig.7. It is believed that higher eccentricity will lead to higher amplitude of each side lobe and more harmonic distortions.

No difference was observed between the two IASK signals in frequency-domain, with offset inclination angles equal to $\pi / 4$ and $-\pi / 4$ respectively.

\section{8-Elliptical Phase Shift Keying}

For its capability of 3-bit information transmission under the same carrier frequency, 8-EPSK was selected as a typical application to illustrate the signal characteristics of EPSK.

Known from its definition, 8-EPSK can be regarded as the combination of QPSK based on elliptical carrier and IASK. Thus, evaluations of 8-EPSK can also be accomplished through combining the analyses of these two modulation methods.

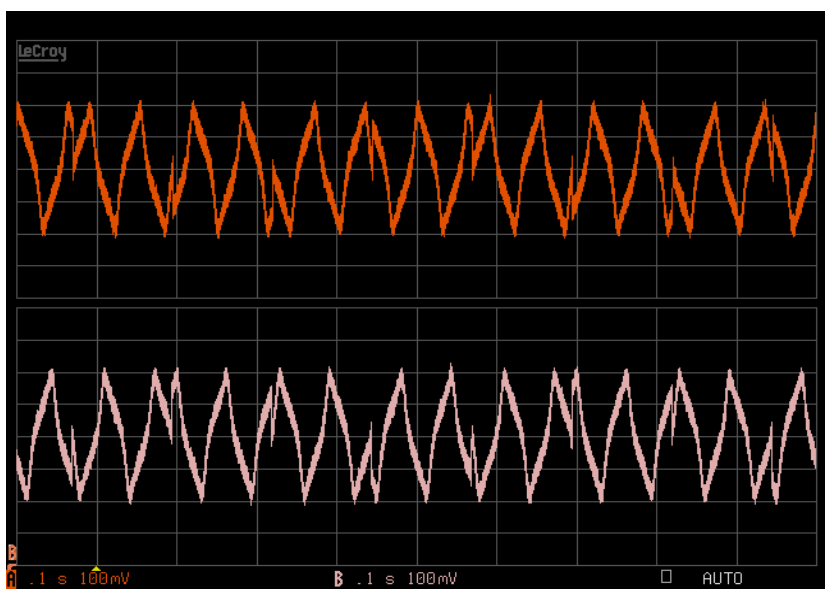

Figure 8. $\quad$ 8-EPSK signals at eccentricity of 0.9 
Once the value of eccentricities were fixed, shape of 8-EPSK waveform was observed to be identical with that of IASK waveform (combination of $\pm \pi / 4$ IASK signals), except the phase jump between each pair of signals (Fig.8). Therefore, it's believed that characteristics of 8-EPSK signals are quite similar with those of IASK signals.

In detection, distinction between the pair of IASK signals can be employed to decide the first bit of received 3-bit message sequence, while the phase jump between each pair of signals can be used for phase matching. Thus analysis result of 8-EPSK signal characteristics have contributed to the following detection method: upon receiving the signal, the first bit can be determined by detecting which ellipse has been used to send out the first bit information; and then the last two bits can be detected by identifying which quadrant the incoming signal vector lies in.

\section{BER CHARACTERISTICS OF IASK, 4-EPSK AND 8-EPSK}

In this part, BER performance comparisons of IASK vs. BPSK, 4-EPSK vs. QPSK and 8-EPSK vs. 8PSK modulation methods under one-path Rayleigh fading environment [2], and BER characteristic comparisons of these three newly proposed modulation methods under AWGN and under one-path Rayleigh fading, have been accomplished through Matlab simulation.

Detection of IASK signal was accomplished through comparing the waveform similarities of received signal and four reference signals, or, in other words, by using correlation detection [3].

As an instance, two ellipses inclined at $\pi / 4$ and $-\pi / 4$ were used as the transmission carrier signals. Thus, expression of the received noise-corrupted signals $r(t)=s( \pm \pi / 4, t)+n(t)$ can be obtained from Equation (3) as:

$$
r(t)=a \sqrt{\frac{1-e_{c}{ }^{2}}{1-e_{c}^{2} \cos ^{2}\left(w_{r} t \pm \frac{\pi}{4}\right)}} \cos \left(\omega_{r} t\right)+n(t)
$$

To discriminate the received elliptical signals, four noisefree elliptical signals have been used as references. Their expressions were given out as follows:

$$
\begin{gathered}
S_{\sin }\left( \pm \frac{\pi}{4}, t\right)=a \sqrt{\frac{1-e_{c}{ }^{2}}{1-e_{c}{ }^{2} \cos ^{2}\left(w_{r} t \pm \frac{\pi}{4}\right)}} \sin \left(\omega_{r} t\right) \\
S_{\cos }\left( \pm \frac{\pi}{4}, t\right)=a \sqrt{\frac{1-e_{c}{ }^{2}}{1-e_{c}{ }^{2} \cos ^{2}\left(w_{r} t \pm \frac{\pi}{4}\right)}} \cos \left(\omega_{r} t\right)
\end{gathered}
$$

Correlations between reference signals and the received signal determine the waveform similarity or dissimilarity between each pair of signals, and which can be calculated as:

$$
\begin{gathered}
c_{\cos }\left( \pm \frac{\pi}{4}, t\right)=r(t) \times s_{\cos }\left( \pm \frac{\pi}{4}, t\right) \\
c_{\sin }\left( \pm \frac{\pi}{4}, t\right)=r(t) \times s_{\sin }\left( \pm \frac{\pi}{4}, t\right)
\end{gathered}
$$

Then within one sampling period $\mathrm{T}$,

$$
\begin{gathered}
C_{C O S}\left( \pm \frac{\pi}{4}, T\right)=\sum_{t_{0}}^{t_{0}+T} c_{\cos }\left( \pm \frac{\pi}{4}, t\right) \\
C_{S I N}\left( \pm \frac{\pi}{4}, T\right)=\sum_{t_{0}}^{t_{0}+T} c_{\sin }\left( \pm \frac{\pi}{4}, t\right)
\end{gathered}
$$

Signals' envelopes were squared, and the received signal was determined corresponding to the larger decision variable:

$$
\begin{gathered}
D_{\pi / 4}=\left(C_{C O S}\left(\frac{\pi}{4}, t\right)\right)^{2}+\left(C_{S I N}\left(\frac{\pi}{4}, t\right)\right)^{2} \\
D_{-\pi / 4}=\left(C_{C O S}\left(-\frac{\pi}{4}, t\right)\right)^{2}+\left(C_{S I N}\left(-\frac{\pi}{4}, t\right)\right)^{2}
\end{gathered}
$$

If $D_{\pi / 4}>D_{-\pi / 4}$, envelope detector judges that the ellipse inclined at $\pi / 4$ has been used to send out the signal;

If $D_{-\pi / 4}>D_{\pi / 4}$, envelope detector judges that the ellipse inclined at $-\pi / 4$ has been used to send out the signal.

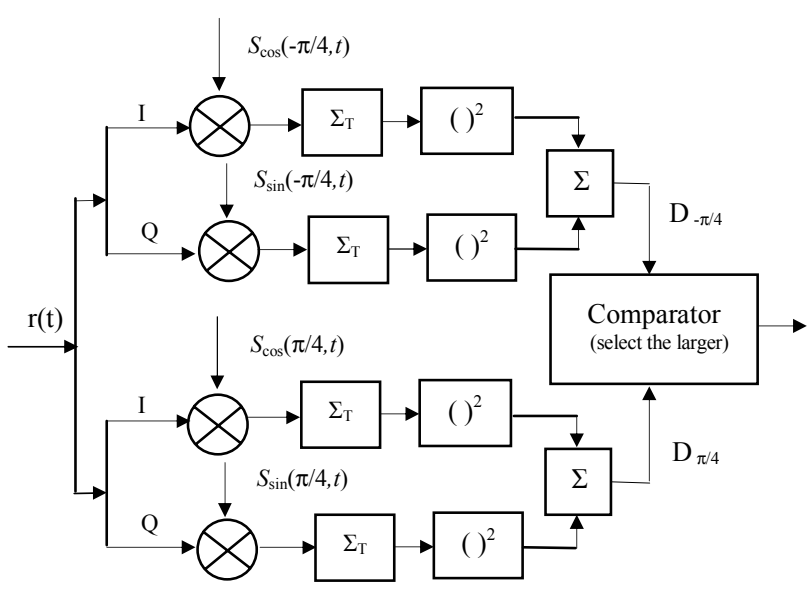

Figure 9. Noncoherent detection of IASK signals 
To demonstrate effect of eccentricity to the detection result, eccentricity was set to be a changing variable from 0.1 to 0.8 in IASK simulation. The graph showed that detection result was very sensitive to the eccentricity, BER performance improved quickly as centricity was increased from 0.1 to 0.8 (Fig.10).

As detection of 4-EPSK signals was quite similar with that of 8-EPSK, this paper only gave out the demodulation of 8EPSK signals.

As mentioned when defining 8-EPSK in section 2.4, 8-EPSK can be regarded as combination of QPSK based on elliptical carrier and IASK, so detection of the first bit can be accomplished by using the same method as that used in IASK. As the increase in eccentricity can contribute to the improvement of IASK performance, hence contribute to the better performance of 8-EPSK in terms of BER, two IASK carrier signals of having eccentricity 0.9 have been selected in simulation. Thus, horizontal and vertical parts of the signals can be expressed as:

$$
\begin{aligned}
& S_{\cos }\left( \pm \frac{\pi}{4}, t\right)=a \sqrt{\frac{1-e_{c}^{2}}{1-e_{c}^{2} \cos ^{2}\left(\omega_{r} t \pm \frac{\pi}{4}\right)}} \cos \left(\omega_{r} t+\phi_{j}\right) \\
& S_{\cos }\left( \pm \frac{\pi}{4}, t\right)=a \sqrt{\frac{1-e_{c}^{2}}{1-e_{c}^{2} \cos ^{2}\left(\omega_{r} t \pm \frac{\pi}{4}\right)}} \sin \left(\omega_{r} t+\phi_{j}\right)
\end{aligned}
$$

Where $j=1,2,3,4$, and $\phi_{j}$ shifts phase of waveform to one of four states.

Then in constellations of 8-EPSK, signals can be given out as:

$$
s(t)=s_{\cos }\left( \pm \pi / 4, t_{0}\right)+j \times s_{\cos }\left( \pm \pi / 4, t_{0}\right)
$$

Real part and imagine part of signals are transmitted in I and Q channels respectively.

After detection of the first bit, last two bits of the message sequences then can be detected by identifying which quadrant the incoming signal vector lies in. Decision range of phase matching for each message sequence is identical with each quadrant in the constellation, which is twice of that in traditional 8PSK.

Suppose that the ellipse inclined at $\pi / 4$ has been detected to be used to send out the signal (the first bit is binary number 0 ), then received signal $r\left(\pi / 4, t_{0}\right)$ can be expressed in constellations as:

$$
r\left(\frac{\pi}{4}, t_{0}\right)=\left(r_{\cos }\left(\frac{\pi}{4}, t_{0}\right)+j \cdot r_{\text {sin }}\left(\frac{\pi}{4}, t_{0}\right)\right)
$$

In Matlab simulation, phase of the received signal in constellations can be calculated directly by using built-in function "angle" as:

$$
\text { ang } \_ \text {pha }=\operatorname{angle}\left[r_{\cos }\left(\pi / 4, \mathrm{t}_{0}\right)+\mathrm{j} \cdot r_{\sin }\left(\pi / 4, \mathrm{t}_{0}\right)\right] \text {; }
$$

Please note that ang pha lies between $\pm \pi$, and accordingly four decision thresholds should be made as: $[-\pi,-\pi / 2),[-\pi / 2,0),[0$, $\pi / 2$ ) and $[\pi / 2, \pi)$. Without a loss of generality, ang pha was assumed to lie inside $[-\pi / 2,0)$, the received message sequence then was determined as [011].

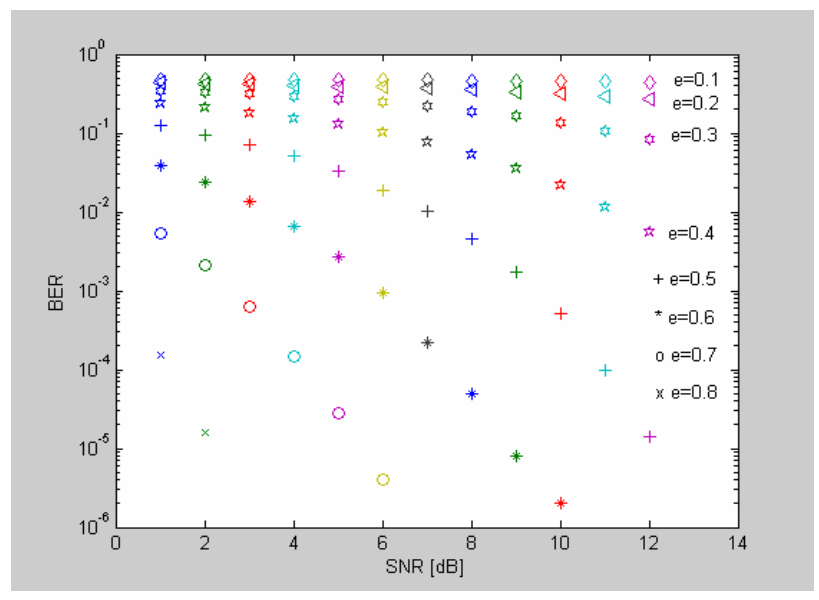

Figure 10. BER performance of IASK

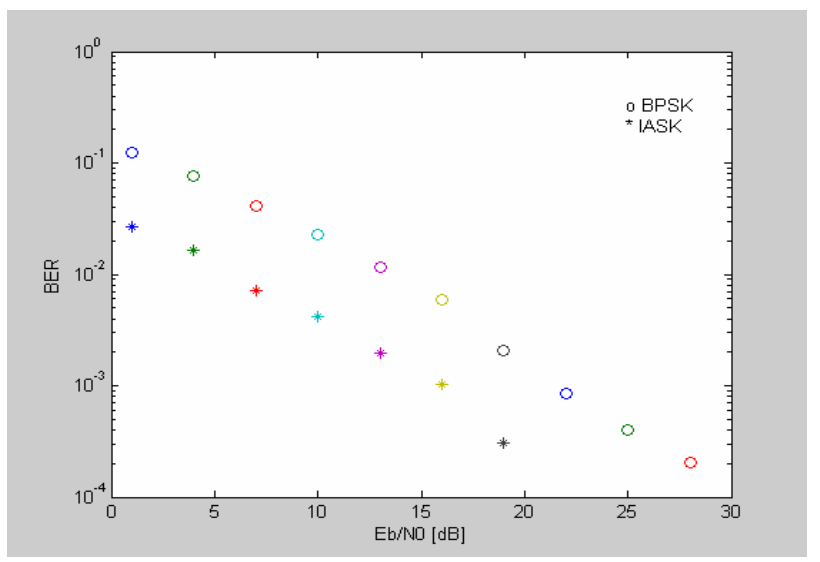

Figure 11. Performance comparison between IASK and BPSK under one-path Rayleigh fading

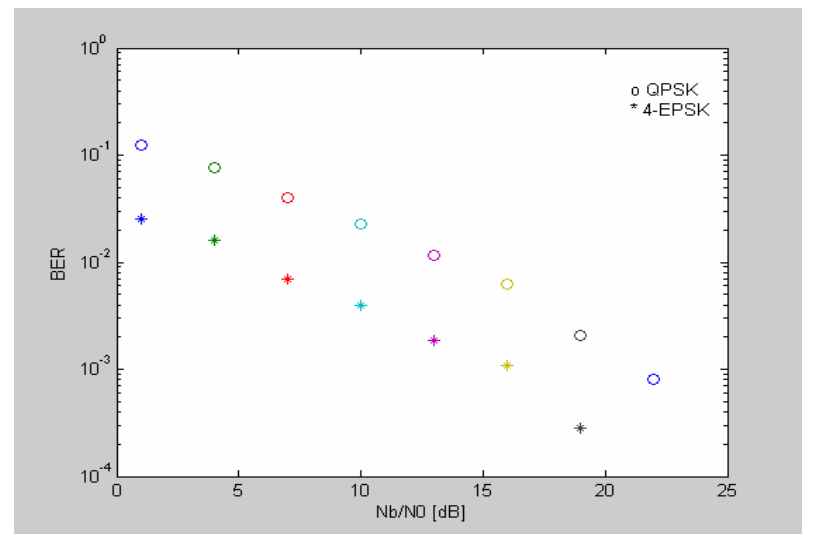

Figure 12. Performance comparison between QPSK and 4-EPSK under one-path Rayleigh fading 


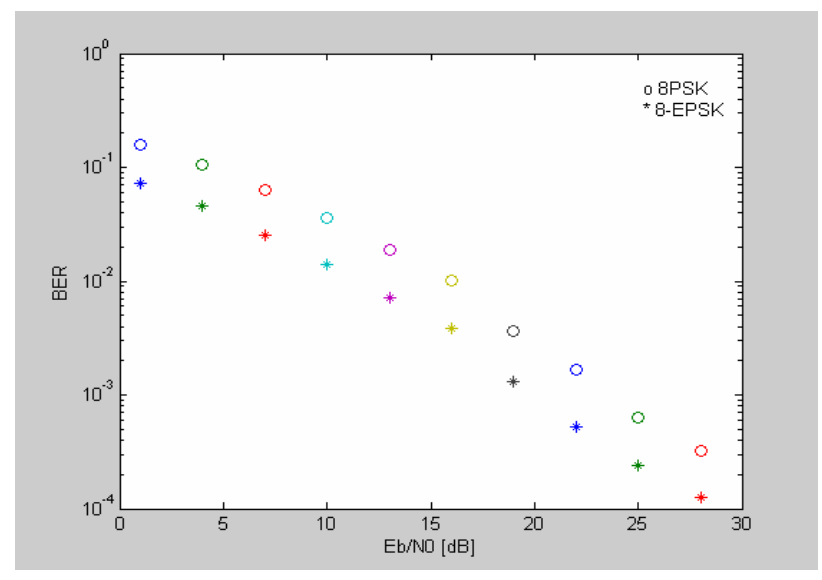

Figure 13. Performance comparison between 8PSK and 8-EPSK under one-path Rayleigh fading

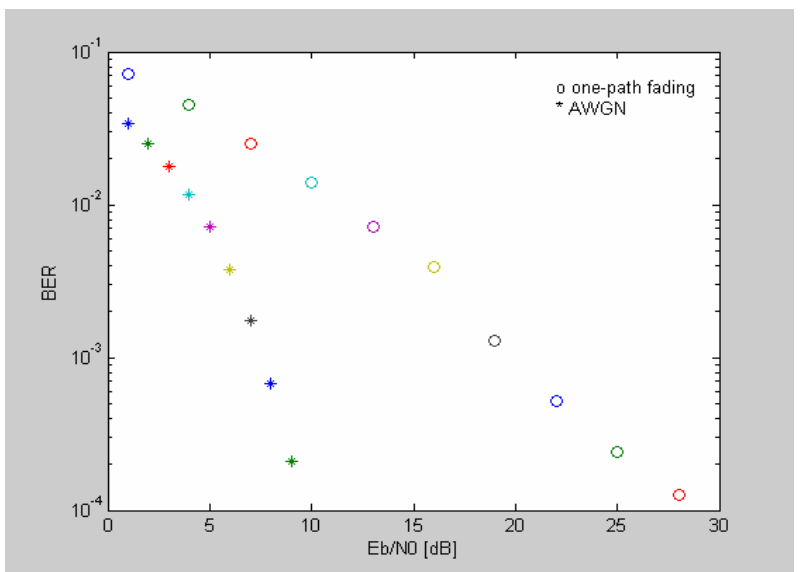

Figure 14. 8-EPSK BER Performance comparison under AWGN and under one-path Rayleigh fading

As modulation methods capable of 1-bit, 2-bit and 3-bit information transmission under the same carrier frequency respectively, IASK, 4-EPSK and 8-EPSK have been compared with BPSK, QPSK and 8PSK respectively through Matlab simulation. In all these three cases, newly proposed modulation schemes have shown obvious advantages over existing modulation methods in terms of BER (Fig.11, Fig.12 \& Fig.13).

BER performance comparison under AWGN and under one-path Rayleigh fading have also been carried out for the newly proposed three modulation schemes, characteristics shown in the result are quite similar with those of traditional modulation scheme (Fig.14).

\section{CONCLUSIONS}

Some modulation methods based on Elliptical Modulation Scheme have been evaluated in this paper, namely are Eccentricity Shift Keying, Inclination Angle Shift Keying and Elliptical Phase Shift Keying. Signal characteristics of these three modulation schemes have been demonstrated and analyzed, through observing real signals generated by using DSPs, both in time-domain and frequency-domain. IASK, 4-EPSK and 8-EPSK have shown advantages over existing modulation schemes in terms of BER through Matlab simulation, and two modulation methods in each comparison pair have the same transmission capability. Effects of eccentricity to both signal and simulation result can be concluded as: the larger the eccentricity, the better the BER, and more harmonics in power spectrum.

To investigate bandwidth efficiency characteristics of our newly proposed modulation methods in comparison with existing modulation methods, and the trade-off relationship between BER performance and bandwidth efficiency, we are going to design the optimum filters for IASK, 4-EPSK and 8-EPSK; to verify both BER and bandwidth efficiency characteristics of these three modulation methods in real communication environment, we will carry out experiments by using some wireless communication equipments; investigation of the possibility of developing new modulation schemes through combining all kinds of modulation variables introduced by Elliptical Modulation, and all kinds of existing modulation methods, will also be scheduled.

\section{ACKNOWLEDGMENT}

This research project is supported by Japanese Monbukagakusho Grants-in-Aid for Scientific Research, Exploratory Research NO.15656094.

\section{REFERENCES}

[1] S.Shimamoto, "Proposal of New Modulation Scheme Employing Elliptical Circle". IEEE Wireless Communications and Networking Conference 2003. March 2003, New Orleans, USA.

[2] Hiroshi Harada and Ramjee Prasad, Simulation and Software Radio. London: Artech House, 2002.

[3] B.P.Lathi, "Binary Differential PSK Example," in Digital Communications-Fundamentals and Applications ( Second Edition ), pp.196-200, Prentice Hall, New Jersey, 2001. 